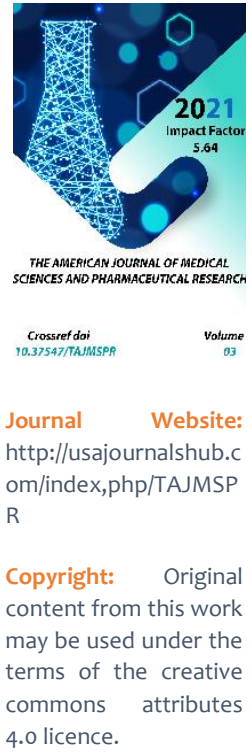

\title{
Comparative Characteristics Of Ferrokinetic Parameters And Their Relationship With Inflammation In Patients With CHF In Advanced And Senile Age
}

\author{
Akhmedova N.Sh \\ Bukhara State Medical Institute, Uzbekistan \\ Abdullaeva U.K. \\ Bukhara State Medical Institute, Uzbekistan \\ Musulmanova D.F. \\ Bukhara State Medical Institute, Uzbekistan
}

\section{ABSTRACT}

To perform a comparative analysis of anemia of chronic disease (ACD) and iron-deficiency anemia (IDA) in late middle-aged and elderly patients with chronic heart failure (CHF) by ferrokinetic parameters, inflammation indexes, and their associations. Materials and methods. 65 patients with ischemic heart disease were evaluated, including 35 patients with $\mathrm{CHF}$ and $A C D, 10$ patients with CHF and IDA, and 20 patients without CHF, ACD, and IDA (control group, CG). Results. Patients with CHF and IDA had true iron deficiency whereas $54 \%$ of patients with CHF and ACD had functional iron deficiency, and $46 \%$ of patients had no iron deficiency. Levels of acute phase proteins, ferritin and hepcidin, C-reactive protein (CRP), and interleukin-6 (IL-6) were highly significantly different in patients with $\mathrm{CHF}$ and $\mathrm{ACD}$ and patients with $\mathrm{CHF}$ and IDA; positive and significant correlations were found for levels of IL- 6 and ferritin, IL- 6 and CRP, and CRP and hepcidin. In patients with CHF and IDA, levels of acute phase proteins, ferritin and hepcidin, CRP, and IL- 6 were low and correlations of IL- 6 with ferritin, IL- 6 with CRP, and CRP with hepcidin were non-significant. Concentrations of erythropoietin were significantly higher in patients with CHF and ACD and patients with CHF and IDA compared to the control group; however, significant differences between them were absent.

\section{KEYWORDS}

Chronic heart failure, anemia of chronic disease, iron deficiency anemia, hepcidin, ferritin, erythropoietin, interleukin-6 (IL-6) 


\section{INTRODUCTION}

Anemia in patients with CHF occurs in $4-53 \%$ of cases [1], which depends on the severity of the underlying disease and the criteria used for anemia [2]. Especially often (up to $80 \%$ ) it develops in patients with FC IV CHF [3]. In a large systematic review of several studies, which included 153180 patients with CHF, 48\% mortality was noted within six months in patients with anemia compared with $29.5 \%$ without anemia [4]. In the RENAISSANCE study [5], it was found that an increase in hemoglobin level by $1 \mathrm{~g} / \mathrm{dL}$ in CHF patients reduces the risk of death by $15.8 \%$. A particularly unfavorable effect of anemia on the course of $\mathrm{CHF}$ and an increase in the risks of cardiovascular events was shown in the TIME study [6] in patients over 75 years of age. In this age group of patients, a decrease in hemoglobin by $1 \mathrm{~g} / \mathrm{dl}$ led to an increase in the risk of death from cardiac causes by $28 \%$, the risk of developing acute myocardial infarction, ACS, and the need for percutaneous coronary interventions by $23 \%$ [6].

In $\mathrm{CHF}$, the 2 most common forms of anemia most often develop: iron deficiency (IDA), which occurs in up to $21 \%$ of cases [7], and anemia of chronic diseases (ACD), the frequency of which is up to $58 \%$ of cases [8].

Unlike IDA, in which the diagnosis, as a rule, is not difficult, ACD is often not diagnosed [9], primarily due to the fact that in the early stages it proceeds as normocytic and normochromic [10], and a moderate decrease in values hemoglobin is explained by an alimentary factor, while its manifestations such as shortness of breath, weakness and tachycardia fit the doctors entirely within the framework of CHF. It is known that a clinical blood test cannot objectively indicate iron deficiency in patients with anemia [10]. Meanwhile, it was found that in patients with ACD and IDA, iron deficiency is different in severity: with IDA true, and with ACD - functional or "redistribution" and for its detection there are not enough hemogram indicators, but it is necessary to study ferrokinetic indices, first of all, ferritin [ 11, 12], which, unlike IDA in ACD, remains normal or, more often, elevated, since, like C-reactive protein (CRP), it is an acute phase of inflammation protein synthesized in the liver in response to inflammatory stimuli [13]. It is also important to study other indicators of ferrokinetics: hepcidin, which also has properties characteristic of acute phase proteins, increases in inflammatory processes and which in the pathogenesis of ACD acts as a direct mediator that has a significant effect on iron homeostasis [14]; erythropoietin (EPO), which, according to the literature, increases during IDA, which is stimulated by chronic blood loss [15]. At the same time, the data on the level of EPO in patients with ACD against the background of CHF are contradictory, since some researchers point to an increased level of it $[16,17]$, and others - to a lower one, attributing this to the action of proinflammatory cytokines that negatively affect the production of EPO $[18,19]$. All these issues have not been sufficiently studied in elderly and senile patients with $\mathrm{CHF}$, in whom the identification of the cause of anemia due to high comorbidity is especially difficult, and the management strategy of these patients is fundamentally different. All of the above determines the relevance of this work.

The aim of the study was to compare AChD and IDA in terms of ferrokinetics and to investigate their relationship with inflammation in elderly and senile CHF patients.

\section{MATERIAL AND METHODS}

We examined 65 elderly and senile patients of the Bukhara regional cardiological dispensary with coronary artery disease. Of these, 35 patients (19 women, 16 men) aged 76 to 97 years with $C H F$ and $A C D$; 10 patients ( 6 women, 4 men) aged 76 to 92 years with CHF and IDA; 20 patients ( 11 women, 9 men) aged 77 to 91 
years without $\mathrm{CHF}, \mathrm{ACD}$ and IDA made up the control group (CG).

Since until now there are no official recommendations on the target level of hemoglobin in $\mathrm{CHF}$, and most researchers agree on the value of $12 \mathrm{~g} / \mathrm{dL}$, and also due to the absence of physiological iron losses in women in old and old age, patients were included in the CHF group with ACD. with a hemoglobin level less than $12 \mathrm{~g} / \mathrm{dl}$, with a normal or increased ferritin level (>30 $\mu \mathrm{g} / \mathrm{I})$, and in the CHF group with IDA - patients with a hemoglobin level less than $12 \mathrm{~g} / \mathrm{dl}$, a low ferritin level $(<30 \mu \mathrm{g} / \mathrm{I})$ and proven chronic blood loss (hemorrhoids, erosive gastroduodenitis, gastric ulcer). The control group (CG) included patients with a hemoglobin level of $12 \mathrm{~g} / \mathrm{dL}$ or more, a normal ferritin level (30-100 $\mu \mathrm{g} / \mathrm{L}$ ) and no signs of chronic blood loss. The severity of CHF was assessed according to the CHF classification of the OSSN, 2002. The severity of anemia was assessed by the $\mathrm{WHO}$ classification, 2008. The severity of chronic kidney disease (CKD) was assessed according to the Kidney Disease Outcome Quality Initiative classification (K / DOQI, 2009). Ferritin, transferrin, CRP were studied in blood serum by immunoturbidimetric method. Hepcidin, EPO and interleukin-6 (IL6) were investigated quantitatively in blood serum by enzyme-linked immunosorbent assay. The percentage of transferrin saturation with iron was calculated using the formula: serum iron $(\mu \mathrm{mol} / \mathrm{L}) \times 3.98$ / transferrin ( $\mathrm{g} / \mathrm{L})$.

Exclusion criteria: megaloblastic, hemolytic, aplastic anemias, autoimmune, oncological diseases.

Statistical data processing was performed using the statistical software package "STATISTICA - 8". The probability of a random difference in mean $p$ for 2 groups was determined by Student's $t$ test $(t)$, for a larger number of groups using one-way ANOVA followed by post-hoc Newman-Kells and Duncan tests.

The probability of a random difference in the sums of the ranks of observations was determined for two groups using the MannWhitney test $(U)$, for 3 or more - using the Kruscull-Wallis rank analysis of variance $(\mathrm{H})$, followed by the multiple comparisons test. The significance of the difference in the frequency of occurrence of the traits was determined by Fisher's exact test (TCF) or the $\chi^{2}$ test. Spearman's rank correlation coefficient (S) was used to assess the degree of monotone significant relationship. Differences were considered significant at $p<0.05$.

\section{RESULTS}

It was revealed that patients with $\mathrm{CHF}$ with $A C D$ did not significantly differ from patients with CHF with IDA in terms of age, sex, but significantly differed in the manifestations and course of IHD, the severity of CHF and CKD, since patients with IDA were selected purposefully without inflammatory diseases, without congestive $\mathrm{CHF}$, with minimal comorbidity to exclude the influence of any inflammation on the formation of anemia, but with proven chronic blood loss.

Comparative characteristics of patients with CHF with ACD and CHF with IDA according to hemogram parameters

It was revealed that patients with $\mathrm{CHF}$ with $A C D$ did not significantly differ from patients with CHF with IDA in terms of hemogram indicators: the level of decreased hemoglobin $(\mathrm{Hb})-10.11 \pm 0.223$ and $9.90 \pm 0.46 \mathrm{~g} / \mathrm{dL}$, respectively $(p=0.582)$ and the number of erythrocytes $-3.67 \pm 0.085$ and $3.77 \pm 0.097$ * $1012 / L$, respectively ( $p=0.445)$, but at the same time significantly differed in color index $(C P)-0.85 \pm 0.014$ and $0.76 \pm 0.03$, respectively ( $p<0.0001)$, the average erythrocyte volume 
$(\mathrm{MCV})-83.86 \pm 0.969$ and $77.50 \pm 1.92 \mathrm{fl}$, respectively $(\mathrm{p}<0.0001)$ and the average hemoglobin content in one erythrocyte $(\mathrm{MCH})$ $-28,76 \pm 0.340$ and $27.21 \pm 1.43 \mathrm{pg}$, respectively $(p=0.027)$ (Table 2$)$. In this regard, patients with normocytic anemia - 71\% and normochromic anemia - 60\% prevailed in the group of $C H F$ with $A C D$, while in the group of CHF with IDA with microcytic anemia - $60 \%$ and hypochromic anemia - $80 \%$.

Comparative characteristics of patients with CHF with ACD and CHF with IDA in terms of ferrokinetics

Significant differences in ferrokinetic parameters were revealed between $\mathrm{CHF}$ patients with ACD and CHF with IDA: ferritin level $-131.97 \pm 17.993$ versus $18.90 \pm 3.59 \mu \mathrm{g} / \mathrm{I}$ ( $p<0.0001$ ), while $54 \%$ of patients showed normal (30-100 $\mu \mathrm{g} / \mathrm{L}$ ) ferritin level, which indicates functional iron deficiency [20, 21], and in $46 \%$ of patients - increased ferritin level (100$505 \mu \mathrm{g} / \mathrm{L}$ ), which indicates the absence of iron deficiency [20, 21], as well as serum iron - 7.30 \pm 0.270 versus $4.86 \pm 0.77 \mu \mathrm{mol} / \mathrm{L}(\mathrm{p}=0.045)$, hepcidin $-23.81 \pm 3.625$ versus $6.31 \pm 1.15 \mathrm{pg} / \mathrm{ml}$ $(p=0.001)$, transferrin $-2.44 \pm 0.084$ versus $2.96 \pm 0.23 \mathrm{~g} / \mathrm{L}(\mathrm{p}<0.0001)$ and the percentage of transferrin saturation with iron - $12.37 \pm$ 0.674 versus $7.22 \pm 1.504 \%(p=0.016)$. Both in patients with $C H F$ with $A C D$ and in patients with CHF with IDA, increased levels of EPO were found without significant differences between them: $44.92 \pm 5.367$ versus $44.13 \pm$ $6.23 \mathrm{mlU} / \mathrm{ml}(\mathrm{p}=0.913)$. However, the EPO level was significantly higher in CHF patients with ACD than in CG patients - $44.92 \pm 5.367$ versus $17.30 \pm 3.302 \mathrm{mlU} / \mathrm{ml}(\mathrm{p}<0.001)$, as well as in CHF patients with IDA than in CG patients $-44.13 \pm 6.23$ versus $17.30 \pm 3.302 \mathrm{mlU} / \mathrm{ml}$ (p $<0.001)$.

Comparative characteristics of patients with CHF with ACD and CHF with IDA in terms of inflammation
Significant differences in CRP level were revealed between $C H F$ patients with $A C D$ and CHF with IDA $-20.6 \pm 5.3$ versus $5.58 \pm 1.25 \mathrm{mg}$ / I $(p=0.049)$. Also, the CRP level was significantly higher in CHF patients with $A C D$ than in CG patients $-20.6 \pm 5.3$ versus $2.9 \pm 0.3$ $\mathrm{mg} / \mathrm{I}(\mathrm{p}=0.044)$. There were no significant differences in CRP levels between CHF patients with IDA and CG patients $-5.58 \pm 1.25$ versus 2.9 $\pm 0.3 \mathrm{mg} / \mathrm{I}(\mathrm{p}=0.769)$. In addition, significant differences in the level of IL- 6 were revealed between patients with $\mathrm{CHF}$ with $\mathrm{ACD}$ and $\mathrm{CHF}$ with IDA $-5.51 \pm 0.783$ versus $2.23 \pm 0.99 \mathrm{pg} / \mathrm{ml}$ $(p=0.011)$, as well as between patients with CHF with ACD and CG patients - $5.51 \pm 0.783$ versus $0.82 \pm 0.13 \mathrm{pg} / \mathrm{ml}(p=0.001)$, while there were no significant differences between CHF patients with IDA and CG patients $-2.23 \pm$ 0.99 versus $0.82 \pm 0.13 \mathrm{pg} / \mathrm{ml}(\mathrm{p}=0.265)$.

Correlation of ferrokinetic indices with inflammation indices in CHF patients with $A C D$, CHF with IDA and CG patients

During the Spearman correlation analysis in CHF patients with $A C D$, significant positive relationships of average strength were revealed: between IL- 6 and ferritin $(r(S)=$ $0.404, p(r)=0.016), I L-6$ and EPO $(r(S)=0.424$, $p(r=0.011), I L-6$ and $\operatorname{CRP}(r(S)=0.427, p(r)=$ $0.010)$, CRP and hepcidin ( $r(S)=0.561, p(r)$ $<0.0001$ On the contrary, insignificant relationships were found in $\mathrm{CHF}$ patients with IDA: between IL- 6 and ferritin $(r(S)=0.193, p$ $(r)=0.593)$, IL-6 and EPO $(r(S)=-0.006, p(r)=$ $0.986)$, IL-6 and CRP $(r(S)=0.623, p(r)=0.071)$, CRP and hepcidin $(r(S)=-0.498, p(r)=0.143)$. $C G$ patients also revealed insignificant relationships: between IL- 6 and ferritin $(r(S)=$ 0.106, $p(r)=0.657)$, IL-6 and EPO ( $r(S)=0.056$, $p(r)=0.816)$, IL-6 and CRP $(r(S)=0.387, p(r)=$ $0.092), C R P$ and hepcidin $(r(S)=0.008, p(r)=$ 0.972). (Fig. 1: A, B, C , D).

Thus, between elderly and senile CHF patients with ACD and CHF with IDA of elderly and senile 
age, significant laboratory differences were revealed, consisting, first, in different severity of iron deficiency (true with IDA and functional or "redistributive" deficiency with ACD or its absence), at the same time, in comparable increased levels of EPO, however, with different mechanisms of its increase; secondly, the identification of high levels of ferrokinetic parameters (ferritin, hepcidin and EPO) in patients with CHF with $A C D$, as well as high levels of inflammation indicators: CRP and IL-6 and significant positive relationships between them, and, on the contrary, the identification of patients with CHF with IDA of low levels of ferrokinetic indices (ferritin and hepcidin), as well as low levels of inflammation indices: CRP and IL- 6 and the absence of links between them.

\section{DISCUSSION}

It is known that hemogram indices make it possible to conclude that anemia is present, but it is not possible to determine its form and severity of iron deficiency [11]. At the same time, the study of the main indicators of ferrokinetics provides more comprehensive information: it allows one to judge the presence and severity of iron deficiency, to establish a connection between anemia and inflammation. According to the data obtained in CHF patients with IDA, a true iron deficiency was revealed: extremely low levels of serum iron, ferritin and the percentage of transferrin saturation with iron. Whereas in CHF patients with ACD moderately reduced levels of serum iron and the percentage of transferrin saturation with iron were revealed, while 54\% of patients had a normal level of ferritin, which indicates a functional or "redistribution" iron deficiency [20, 21], and $46 \%$ patients - an increased level of ferritin, which indicates the absence of iron deficiency [20, 21].

Noteworthy is the significantly increased level of EPO both in patients with $A C D$ and in patients with IDA relative to patients in CG. It is known that chronic blood loss in IDA increases the synthesis of EPO [15], and in ACD many authors, when describing the mechanisms of its development, indicate a decrease in EPO production under the influence of proinflammatory cytokines $[18,19]$. However, a number of authors believe that EPO synthesis increases in patients with $\mathrm{CHF}$ due to a decrease in renal blood flow caused by the vasoconstrictor effect of neurohormones on renal vessels and the development of hypoxia $[22,23]$. In addition, in CHF, low renal blood flow stimulates the production of renin, and this, in turn, of angiotensin II (AII), and a high level of All leads to an increase in sodium reabsorption, which is accompanied by an increase in the consumption of ATP and oxygen, which ultimately increases the production of EPO [24]. Therefore, in many patients with CHF and ACD, not decreased, but, on the contrary, normal or increased levels of EPO are determined $[16,17]$.

According to our data, in CHF patients with $A C D$, in contrast to CHF patients with IDA and CG patients, high levels of inflammation indicators were revealed: CRP, as well as other indicators, which, like CRP, are proteins of the acute phase of inflammation - rarely used to assess inflammatory syndrome - ferritin and practically not used yet - hepcidin, synthesized in the liver under the action of proinflammatory cytokines $[13,14]$. It is known that hepcidin blocks the release of iron from macrophages and the absorption of iron in the intestine, which ultimately leads to a functional iron deficiency with a sufficient amount in the body, which is characteristic of ACD [25]. The opposite situation occurs in IDA, in which there is a decrease in the expression of hepcidin, which leads to an increase in the uptake of iron from both macrophages and the intestine [14]. Also, in patients with CHF with $A C D$, a highly significant level of cytokine, IL-6, was revealed, 
which was previously shown by other authors in $A C D$ [15].

In connection with the above, it is important to identify significant positive relationships in $\mathrm{CHF}$ patients with ACD between IL- 6 and ferritin, IL6 and EPO, IL- 6 and CRP, CRP and hepcidin, that is, indicators of inflammation and indicators of ferrokinetics, which indicates inflammation as the reason for the formation of $\mathrm{AHZ}$. In contrast, in patients with CHF with IDA, similar relationships between the parameters of ferrokinetics and indicators of inflammation are insignificant.

CONCLUSION. Thus, between elderly and senile CHF patients with ACD and CHF with IDA of elderly and senile age, significant differences in laboratory parameters were revealed, firstly, consisting in different severity of iron deficiency (true with IDA and functional or "redistributive" deficiency with ACD or its absence) and at the same time, the increased levels of EPO are comparable, but with different mechanisms of its increase. Secondly, high levels of ferrokinetic parameters (ferritin, hepcidin and EPO), as well as high levels of inflammation indicators: CRP and IL-6 and significant positive relationships between them, and, on the contrary, in CHF patients with IDA were revealed in patients with $\mathrm{CHF}$ with ACD. revealed low levels of ferrokinetic parameters (ferritin and hepcidin), as well as low levels of inflammation indicators (CRP and IL-6) and the absence of links between them.

All of the above must be taken into account in the differential diagnosis of these two most frequent anemias in elderly and senile CHF patients, since the management strategy of these patients and approaches to treatment are fundamentally different.

\section{REFERENCES}

1. Lazareva N. V., Oshchepkova E. V., Tereschenko S. N. Comorbidity(heart failure according the register). Kardiologicheskij Vestnik. 2016;XI(4):24-31.

2. Tang Y-D. Anemia in Chronic Heart Failure: Prevalence, Etiology, Clinical Correlates, and Treatment Options. Circulation. 2006;113 (20):2454-61. DOI:10.1161/CIRCULATIONAHA. 105.583666

3. van Deursen VM, Urso $R$, Laroche $C$, Damman K, Dahlström U, Tavazzi L et al. Co-morbidities in patients with heart failure: an analysis of the European Heart Failure Pilot Survey: Co-morbidities in heart failure. European Journal of Heart Failure. 2014;16 (1):103-11. DOI:10.1002/ejhf. 30

4. Groenveld HF, Januzzi JL, Damman K, van Wijngaarden J, Hillege HL, van Veldhuisen DJ et al. Anemia and Mortality in Heart Failure Patients. Journal of the American College of Cardiology. 2008;52 (10):818-27. DOI:10.1016/j. jacc. 2008.04.061

5. Anand I. Anemia and Its Relationship to Clinical Outcome in Heart Failure. Circulation. 2004;110 (2):149-54. DOI:10.1161/01. CIR. 0000134279.79571 .73

6. Muzzarelli S, Pfisterer M. Anemia as independent predictor of major events in elderly patients with chronic angina. American Heart Journal. 2006;152 (5):9916. DOI:10.1016/j. ahj. 2006.06.014

7. Anand IS. Anemia and Chronic Heart Failure. Journal of the American College of Cardiology. 2008;52 (7):501-11. DOI:10.1016/j. jacc. 2008.04.044

8. Ezekowitz JA, McAlister FA, Armstrong $\mathrm{PW}$. Anemia is common in heart failure and is associated with poor outcomes: insights from a cohort of 12065 patients with newonset heart failure. Circulation. 2003;107 (2):223-5.

9. Rudakova L. E., Belyaeva Yu. B., Fatkabrarova A. M., Kozina L. E. Anemia in chronic heart failure (according to the cardiology Department). Theoretical and applied aspects of modern science. 2014;62:128-30. 
10. Zubrikhina G. N., Blindar V. N., Matveeva I. I. Differential diagnosis of anemia in true and functional iron deficiency in patients with chronic diseases (malignant tumors). Therapeutic archive. 2016;88(4):61- 7 .

11. [Zubrikhina G. N., Blindar V. N., Matveyeva I. I. The possibilities of modern automated clinical blood analysis in differentiated diagnostic of true and redistributing (functional) iron deficiency under anemic syndrome in oncologic patients. Russian Clinical Laboratory Diagnostics. 2014;59(5:21-5.

12. Stuklov N. I. Iron Deficiency and Anemia in Patients with Chronic Heart Failure. Rational Pharmacotherapy in Cardiology. 2017;13(5):651-60.

13. Koorts $A M$, Viljoen $M$. Ferritin and ferritin isoforms I: Structure - function relationships, synthesis, degradation and secretion. Archives of Physiology and Biochemistry. 2007;113 (1):30-54. DOI:10.1080/13813450701318583

14. Arezes J, Nemeth E. Hepcidin and iron disorders: new biology and clinical approaches. International Journal of Laboratory Hematology. 2015;37:92-8. DOI:10.1111/ijlh. 12358

15. Camaschella C. Iron-Deficiency Anemia. Longo DL, editor. New England Journal of Medicine. 2015;372 (19):1832-43. DOI:10.1056/NEJMra1401038

16. Nagai $T$, Nishimura $K$, Honma $T$, Higashiyama A, Sugano Y, Nakai $M$ et al. Prognostic significance of endogenous erythropoietin in long-term outcome of patients with acute decompensated heart failure: Endogenous erythropoietin in acute heart failure. European Journal of Heart Failure. 2016;18 (7):803-13. DOI:10.1002/ejhf. 537

17. van der Meer P, Lok DJ, Januzzi JL, de la Porte PWB-A, Lipsic E, van Wijngaarden J et al. Adequacy of endogenous erythropoietin levels and mortality in anaemic heart failure patients. European Heart Journal. 2008;29 (12):1510-5. DOI:10.1093/eurheartj/ehn205

18. Blindar V. N., Zubrikhina G. N., Matveeva I. I. Anemic syndrome and main metabolites ferrokinetic (ferritin, soluble transferrin receptor, progestin, hepcidin-25 and endogenous erythropoietin). Medical alphabet. 2015;2(8):16-9.

19. Azerbaijan State Advance Training Institute for Doctors, Baku, Azerbaijan, Zakhidova KK. Correlation Between Concentration of Pathological Cytokines and Erythropoietin in Patients with Chronic Heart Failure with Anemic Syndrome. Annals of the Russian academy of medical sciences. 2014;69 (12):32-7. DOI:10.1569o/vramn. v69i1-2.939

20. Nairz M, Theurl I, Wolf D, Weiss G. Iron deficiency or anemia of inflammation? Differential diagnosis and mechanisms of anemia of inflammation. Wiener Medizinische Wochenschrift. 2016;166 (1314):411-23. DOI:10.1007/s10354-016-0505-7

21. Drozd $M$, Jankowska $E A$, Banasiak $W$, Ponikowski P. Iron Therapy in Patients with Heart Failure and Iron Deficiency: Review of Iron Preparations for Practitioners. American Journal of Cardiovascular Drugs. 2017;17 (3):183-201. DOI:10.1007/s40256016-0211-2

22. Anand IS. Heart failure and anemia: mechanisms and pathophysiology. Heart Failure Reviews. 2008;13 (4):379-86. DOI: 10.1007/s10741-008-9088-8

23. Caramelo C, Justo S, Gil P. Anemia in heart failure: pathophysiology, pathogenesis, treatment, and incognitae. Rev Esp Cardiol. 2007;60 (8):848-60. PMID:17688854

24. Diskin CJ. Erythropoietin, haemoglobin, heart failure, and mortality. European Heart Journal. 2008;29 (21):2695-2695. DOI:10.1093/eurheartj/ehn399

25. Cappellini MD, Comin-Colet J, de Francisco A, Dignass A, Doehner W, Lam CS et al. Iron deficiency across chronic inflammatory 
The American Journal of Medical Sciences and Pharmaceutical Research

(ISSN - 2689-1026)

Published: April 30, 2021 | Pages: 142-149

Doi: https://doi.org/10.37547/TAJMSPR/Volume03Issue04-19

conditions: International expert opinion on definition, diagnosis, and management.

American Journal of Hematology. 2017;92

(10):1068-78. DOI:10.1002/ ajh.24820 